\title{
Article \\ Sodium Intake and Incidence of Diabetes Complications in Elderly Patients with Type 2 Diabetes-Analysis of Data from the Japanese Elderly Diabetes Intervention Study (J-EDIT)
}

\author{
Chika Horikawa ${ }^{1}$, Rei Aida ${ }^{2}$, Shiro Tanaka ${ }^{2}$, Chiemi Kamada ${ }^{3}$, Sachiko Tanaka ${ }^{4}$, Yukio Yoshimura ${ }^{3}$, \\ Remi Kodera ${ }^{5,6}$, Kazuya Fujihara ${ }^{6}$, Ryo Kawasaki ${ }^{7}$, Tatsumi Moriya ${ }^{8}$, Hidetoshi Yamashita ${ }^{9}$, Hideki Ito ${ }^{5}$, \\ Hirohito Sone ${ }^{6}(\mathbb{D})$ and Atsushi Araki ${ }^{5, *}$
}

Citation: Horikawa, C.; Aida, R.; Tanaka, S.; Kamada, C.; Tanaka, S.; Yoshimura, Y.; Kodera, R.; Fujihara, K.; Kawasaki, R.; Moriya, T.; et al. Sodium Intake and Incidence of Diabetes Complications in Elderly Patients with Type 2 Diabetes -Analysis of Data from the Japanese Elderly Diabetes Intervention Study (J-EDIT). Nutrients 2021, 13, 689. https://doi.org/10.3390/nu13020689

Academic Editor: Murielle Bochud

Received: 30 January 2021

Accepted: 17 February 2021

Published: 21 February 2021

Publisher's Note: MDPI stays neutral with regard to jurisdictional claims in published maps and institutional affiliations.

Copyright: (C) 2021 by the authors Licensee MDPI, Basel, Switzerland. This article is an open access article distributed under the terms and conditions of the Creative Commons Attribution (CC BY) license (https:/ / creativecommons.org/licenses/by/ $4.0 /)$.
1 Department of Health and Nutrition, University of Niigata Prefecture Faculty of Human Life Studies, 471 Ebigase, Higashi-ku, Niigata 950-8680, Japan; horikawa@unii.ac.jp

2 Department of Clinical Biostatistics, Graduate School of Medicine, Kyoto University, Yoshida-Konoe-cho, Sakyo-ku, Kyoto 606-8501, Japan; aida.rei.5c@kyoto-u.ac.jp (R.A.); tanaka.shiro.8n@kyoto-u.ac.jp (S.T.)

3 Training Department of Administrative Dietitians, Shikoku University, 123-1 Ebisuno, Furukawa, Ojin-cho, Tokushima 771-1151, Japan; c-kamada@shikoku-u.ac.jp (C.K.); yyoshimura@shikoku-u.ac.jp (Y.Y.)

4 Department of Public Health, Shiga University of Medical Science, Seta Tsukinowa-cho, Otsu, Sihga 520-2192, Japan; sachikot@belle.shiga-med.ac.jp

5 Department of Endocrinology and Metabolism, Tokyo Metropolitan Geriatric Hospital, 35-2 Sakaecho, Itabashi-ku, Tokyo 173-0015, Japan; remi_kokubo@tmghig.jp (R.K.); hideki_ito@tmghig.jp (H.I.)

6 Department of Hematology, Endocrinology, and Metabolism, Yamagata University Faculty of Medicine, 1-757 Asahimachi-dori, Chuoh-ku, Niigata 951-8510, Japan; kafujihara-dm@umin.ac.jp (K.F.); sone@med.niigata-u.ac.jp (H.S.)

7 Department of Vision Informatics, Graduate School of Medicine Faculty of Medicine, Osaka University, Osaka, 2-2 Yamadaoka, Suita 565-0871, Japan; ryo.kawasaki@ophthal.med.osaka-u.ac.jp

8 Health Care Center, Kitasato University, 1-15-1, Kitazato, Minami-ku, Sagamihara-shi 252-0373, Japan; moriy@kitasato-u.ac.jp

9 Department of Ophthalmology and Visual Science, Yamagata University Faculty of Medicine, 2-2-2 Iidanishi, Yamagata-shi 990-8560, Japan; yamasita@med.id.yamagata-u.ac.jp

* Correspondence: aaraki@tmghig.jp; Tel.: +81-03-3964-1141; Fax: +81-03-3964-1982

Abstract: This study investigates the associations between sodium intake and diabetes complications in a nationwide cohort of elderly Japanese patients with type 2 diabetes aged 65-85. Data from 912 individuals regarding their dietary intake at baseline is analyzed and assessed by the Food Frequency Questionnaire based on food groups. Primary outcomes are times to diabetic retinopathy, overt nephropathy, cardiovascular disease (CVD), and all-cause mortality during six years. We find that mean sodium intake in quartiles ranges from $2.5 \mathrm{~g}$ to $5.9 \mathrm{~g} / \mathrm{day}$. After adjustment for confounders, no significant associations are observed between sodium intake quartiles and incidence of diabetes complications and mortality, except for a significant trend for an increased risk of diabetic retinopathy $(p=0.039)$. Among patients whose vegetable intake was less than the average of $268.7 \mathrm{~g}$, hazard ratios (HRs) for diabetic retinopathy in patients in the second, third, and fourth quartiles of sodium intake compared with the first quartile were 0.87 (95\% CI, 0.31-2.41), 2.61 (1.00-6.83), and 3.70 (1.37-10.02), respectively. Findings indicate that high sodium intake under conditions of low vegetable intake is associated with an elevated incidence of diabetic retinopathy in elderly patients with type 2 diabetes.

Keywords: type 2 diabetes; older adults; medical nutritional therapy; sodium intake; diabetes complications

\section{Introduction}

Excessive sodium intake is regarded as a crucial factor related to the development and worsening of diabetes complications, and the importance of reducing dietary sodium intake 
is stated in guidelines for diabetes care [1-3]. However, epidemiological studies on the relationship between sodium intake and the development of diabetes complications are limited. Some previous prospective studies have examined the relationship between sodium intake and mortality in patients with type 1 diabetes [4] and type 2 diabetes-mainly among the middle-aged and young-old-aged [5-7], and results have been inconsistent. In a study of patients with type 1 diabetes, high 24-h urinary sodium excretion was related to the incidence of end-stage renal disease [4]. Our previous study as part of the Japan Diabetes Complications Study (JDCS) showed that high dietary sodium intake was associated with an elevated incidence of cardiovascular disease (CVD) in patients with type 2 diabetes aged 40-70 [6]. In Japan, there are two prospective longitudinal studies, namely, the JDCS mainly targeted toward middle-aged patients with type 2 diabetes $[8,9]$, and the Japanese Elderly Diabetes Intervention Trial (J-EDIT) targeted to elderly patients with type 2 diabetes [10]. These two studies collected nearly the same information about anthropometric and laboratory tests, clinical characteristics, and outcomes for each patient [8-10].

The aging of the world's population is a major contributor to the prevalence of diabetes [11]. According to the report from the International Diabetes Federation in 2019, 136 million people over 65 years old have diabetes [12]. The world's population in 2019 was 7713 million, with 701 million over the age of 65 years [13]; thus, it is estimated that 1 in 5 elderly people have diabetes. Moreover, it is estimated that the percentage of the population over age 65 years could increase from $9.1 \%$ in 2019 to $15.9 \%$ in 2050 [13]. An increase in elderly patients with diabetes is inevitable. Thus, we investigated whether there was an association between sodium intake and the incidence of diabetes complications, including CVD, overt nephropathy, and diabetic retinopathy, as well as all-cause mortality in elderly patients with type 2 diabetes in the large nationwide multicenter cohort of J-EDIT.

\section{Materials and Methods}

\subsection{Study Cohort}

The study was conducted as part of J-EDIT, a multicenter prospective study on the development of macro- and microvascular complications and physical, psychological, and mental prognoses among Japanese patients with type 2 diabetes from outpatient departments in 39 representative hospitals. Eligible for the study were patients with type 2 diabetes aged $65-85$ and $\mathrm{HbA} 1 \mathrm{c} \geq 7.9 \%$ or $\geq 7.4 \%$ with at least one abnormal finding among body mass index (BMI), blood pressure, or serum lipids. HbA1c assays were standardized by the Laboratory Test Committee of the Japan Diabetes Society (JDS) [3], and the National Glycohemoglobin Standardization Program value for HbA1c [14] was calculated by the following formula: $0.25+1.02 \times$ JDS value [15]. A history of myocardial infarction, stroke, cancer, acute or serious illness, aphasia, and severe dementia within six months from enrollment were criteria for exclusion from the study. Details of study design and baseline characteristics of the J-EDIT were described elsewhere [10]. The protocol for the study, which is in accordance with the Declaration of Helsinki and the Ethical Guidelines for Clinical/Epidemiological Studies of the Japanese Ministry of Health Labor and Welfare, received ethical approval from the institutional review boards of all participating institutes. Written informed consent was obtained from all enrolled patients.

A total of 1173 patients who met the eligibility criteria described above were enrolled in the J-EDIT from March 2001 to February 2002, and 912 patients responded to a baseline dietary survey. The follow-up time was six years, and the dropout rate after six years was $8.9 \%$ (104 cases) [10]. In the present study, we grouped patients analyzed according to the following endpoints: CVD, nephropathy, and retinopathy. The CVD group consisted of 651 patients without a history of angina pectoris, myocardial infarction, or stroke. The nephropathy group included 624 patients after those with a baseline albumin excretion rate of $<150 \mathrm{mg} / \mathrm{g}$ creatinine or serum creatine level $>120 \mu \mathrm{mol} / \mathrm{L}$ were excluded. The retinopathy group was comprised of 327 patients after patients were excluded who had retinopathy or a major ocular disease (e.g., glaucoma, cataract, or a history of cataract surgery or vitrectomy). 


\subsection{Dietary Assessment}

The Food Frequency Questionnaire based on food groups (FFQg) at baseline was used to assess nutritional and food intakes. The FFQg elicited information from the study participant on the average intake per week of 29 food groups and 10 kinds of cookery in commonly used units or portion sizes. The completed questionnaire was reviewed by a dietitian with the participant. The FFQg had been externally validated by comparison with dietary records for seven continuous days by 66 individuals aged 19-60 [16]. The average ratio of the estimates obtained by the FFQg against those by the dietary records was $104 \%$, with ratios ranging from $72 \%$ to $121 \%$. The correlation coefficient between the FFQg and dietary records for sodium intake was 0.43 . To calculate the nutrient and food intakes, we used standardized software for population-based surveys and nutrition counseling in Japan (EIYO-KUN v.4.5, manufactured at the site of the Shikoku University Nutrition Database) based on Standard Tables of Food Composition in Japan [17] edited by the Japanese Ministry of Education, Culture, Sports, Science, and Technology.

\subsection{Outcome Measures}

Macroangiopathy endpoints included definite coronary heart disease (angina pectoris or myocardial infarction) or stroke. Coronary heart disease was diagnosed according to criteria defined by the Multinational Monitoring of Trends and Determinants in Cardiovascular Disease (WHO/MONICA) project $[18,19]$. Stroke was defined based on clinical signs of a focal neurological deficit with rapid onset persisting $\geq 24 \mathrm{~h}$ and confirmed by either computed tomography, or magnetic resonance imaging of the brain [20,21]. Confirmation of endpoints was made by a central committee comprised of experts in each complication based on additional data such as a detailed history, sequential changes in ECG and serum cardiac biomarkers, and results of coronary angiography or brain imaging. The endpoint of nephropathy was decided upon according to the development of overt nephropathy (spot urinary albumin excretion $>300 \mathrm{mg} / \mathrm{g}$ creatinine in two consecutive samples). Japanese Diabetes Complication Study method was used to determine the presence of retinopathy, which was classified into five stages: Stage 0 -no retinopathy; stage 1 -dot hemorrhages, hemorrhages or hard exudates; stage 2-soft exudates; stage 3-intraretinal microvascular abnormalities or venous deformities; and stage 4-neovascularization, preretinal proliferative tissues, vitreous hemorrhages or retinal detachment. Development of retinopathy (from Stage 0 in both eyes at the time of registration to any other stage confirmed) in either eye indicated the endpoint of retinopathy. All-cause mortality was an additional endpoint. Details of the definition of each diabetes complication and all-cause mortality were previously published [10].

\subsection{Statistical Analysis}

Patient characteristics were described according to mean \pm SD, median, interquartile range, or percentage. Univariate and multivariate Cox regression analyses were used to estimate the adjusted hazard ratios (HRs) and 95\% Confidence Interval (CI) for each outcome after a six-year follow-up in relation to sodium intake at baseline by conducting a quartile analysis and assigning the lowest quartile of sodium intake as the referent. Multivariate adjusted analyses were conducted with adjustments for age, sex, BMI, HbA1c, diabetes duration, LDL-cholesterol, HDL-cholesterol, log-transformed triglycerides, insulin treatment, current smoker, alcohol intake, energy intake, physical activity, and systolic blood pressure (SBP). We also performed further analyses adjusted for the use of angiotensin II receptor blocker (ARB), angiotensin-converting enzyme (ACE) inhibitors, and calcium channel blockers at baseline in addition to the aforementioned model. Subgroup analyses in the Cox regression analysis were performed using the following groups: Sex (men vs. women), age ( $<75$ years, $\geq 75$ years), duration of diabetes (whether less than mean value or not), $\mathrm{HbA} 1 \mathrm{c}(<8.5 \%$ vs. $\geq 8.5 \%), \mathrm{SBP}(<140 \mathrm{mmHg}$ vs. $\geq 140 \mathrm{mmHg})$, protein intake (whether $<$ mean value or not) and vegetable intake (whether $<$ mean value or not). All $p$-values are two-sided, and the significance level is 0.05 . All statistical analyses and data 
management were conducted at a central data center using SAS ver. 9.4 (SAS Institute Inc., Cary, NC, USA).

\section{Results}

The baseline clinical characteristics of the 912 elderly patients according to quartiles of total sodium intake are shown in Table 1 . Mean sodium intake was $4.1 \mathrm{~g} / \mathrm{day}$. Patients were divided into four groups according to quartiles of mean intakes of sodium as follows: Q1, $2.5 \mathrm{~g}$ /day; Q2, $3.5 \mathrm{~g} /$ day; Q3, $4.4 \mathrm{~g}$ /day; and Q4, $5.9 \mathrm{~g} /$ day. There were no significant differences in BMI, HbA1c, fasting blood glucose, serum lipids, serum creatinine, eGFR, systolic blood pressure, and prevalence of use of oral hypoglycemic agents, antihypertensive agents, and lipid-lowering agents among the quartiles of sodium intake. Patients in the highest quartile of sodium intake had the lowest prevalence of insulin treatment. At baseline, the prevalence of retinopathy was the lowest in those with the highest quartile of sodium intake $(\mathrm{Q} 1,44.7 \%$; Q2, 45.6\%; Q3, 42.1\%; and Q4, 33.8\%, respectively, trend $p=0.013)$.

Table 1. Baseline clinical characteristics of the 912 elderly patients with type 2 diabetes according to quartiles of sodium intake.

\begin{tabular}{|c|c|c|c|c|c|c|c|c|c|c|c|c|c|c|c|c|c|}
\hline & \multicolumn{4}{|c|}{ Q1 $(n=228)$} & \multicolumn{4}{|c|}{$\mathrm{Q} 2(n=228)$} & \multicolumn{4}{|c|}{ Q3 $(n=228)$} & \multicolumn{4}{|c|}{$\mathrm{Q} 4(n=228)$} & \multirow{2}{*}{$\begin{array}{l}\text { Trend } \\
\qquad p\end{array}$} \\
\hline & $n$ & Mean & \pm & SD & $n$ & Mean & \pm & SD & $n$ & Mean & \pm & SD & $n$ & Mean & \pm & SD & \\
\hline Sodium intake (g/day) & 228 & 2.5 & \pm & 0.5 & 228 & 3.5 & \pm & 0.2 & 228 & 4.4 & \pm & 0.3 & 228 & 5.9 & \pm & 0.9 & $<0.001$ \\
\hline Age $(y)$ & 223 & 71.6 & \pm & 4.7 & 228 & 72.3 & \pm & 4.8 & 228 & 71.8 & \pm & 4.8 & 228 & 71.6 & \pm & 4.3 & 0.591 \\
\hline Women (\%) & 223 & 60.1 & $\%$ & & 228 & 59.7 & $\%$ & & 228 & 50.9 & $\%$ & & 228 & 47.4 & $\%$ & & 0.002 \\
\hline Diabetes duration (y) & 221 & 17.8 & \pm & 9.6 & 222 & 15.8 & \pm & 9.8 & 223 & 16.2 & \pm & 9.3 & 227 & 15.8 & \pm & 9.4 & 0.053 \\
\hline $\begin{array}{l}\text { Body mass index } \\
\left(\mathrm{kg} / \mathrm{m}^{2}\right)\end{array}$ & 227 & 23.9 & \pm & 3.3 & 225 & 23.7 & \pm & 3.6 & 226 & 23.8 & \pm & 3.3 & 228 & 24.2 & \pm & 3.6 & 0.242 \\
\hline HbA1c (\% NGSP value) & 228 & 8.5 & \pm & 1.0 & 228 & 8.4 & \pm & 0.9 & 228 & 8.4 & \pm & 0.9 & 228 & 8.4 & \pm & 0.9 & 0.303 \\
\hline $\begin{array}{l}\text { Fasting blood glucose } \\
(\mathrm{mg} / \mathrm{dl})\end{array}$ & 201 & 171.4 & \pm & 55.6 & 201 & 160.2 & \pm & 43.7 & 211 & 171.8 & \pm & 53.0 & 211 & 166.3 & \pm & 47.2 & 0.825 \\
\hline Total cholesterol (mg/dl) & 228 & 203.5 & \pm & 34.9 & 228 & 203.6 & \pm & 37.1 & 228 & 201.5 & \pm & 36.6 & 228 & 202.3 & \pm & 32.5 & 0.591 \\
\hline Triglycerides (mg/dl) & 228 & 141.0 & \pm & 78.8 & 228 & 134.7 & \pm & 129.5 & 228 & 128.6 & \pm & 64.6 & 228 & 129.8 & \pm & 70.4 & 0.137 \\
\hline HDL cholesterol (mg/dl) & 228 & 54.6 & \pm & 17.2 & 225 & 57.1 & \pm & 19.4 & 227 & 57.3 & \pm & 20.5 & 226 & 56.3 & \pm & 15.4 & 0.326 \\
\hline LDL cholesterol (mg/dl) & 225 & 121.0 & \pm & 32.2 & 221 & 120.9 & \pm & 32.4 & 225 & 119.5 & \pm & 31.4 & 224 & 120.3 & \pm & 28.2 & 0.704 \\
\hline $\begin{array}{l}\text { Systolic blood pressure } \\
(\mathrm{mmHg})\end{array}$ & 228 & 135.7 & \pm & 15.3 & 228 & 138.1 & \pm & 15.4 & 228 & 137.0 & \pm & 16.4 & 228 & 135.0 & \pm & 16.2 & 0.511 \\
\hline $\begin{array}{l}\text { Diastolic blood pressure } \\
(\mathrm{mmHg})\end{array}$ & 228 & 74.0 & \pm & 9.4 & 228 & 75.3 & \pm & 10.4 & 228 & 75.5 & \pm & 9.3 & 227 & 74.7 & \pm & 9.5 & 0.417 \\
\hline Serum creatinine $(\mathrm{mg} / \mathrm{dl})$ & 227 & 0.8 & \pm & 0.3 & 226 & 0.8 & \pm & 0.4 & 225 & 0.9 & \pm & 0.5 & 228 & 0.8 & \pm & 0.2 & 0.546 \\
\hline $\begin{array}{l}\text { eGFR }\left(\mathrm{mL} / \mathrm{min} / 1.73 \mathrm{~m}^{2}\right) \\
\text { Treatment }\end{array}$ & 226 & 65.3 & \pm & 20.3 & 226 & 65.0 & \pm & 18.9 & 224 & 66.3 & \pm & 19.1 & 228 & 67.4 & \pm & 18.3 & 0.195 \\
\hline Treated by insulin (\%) & 227 & 37.0 & $\%$ & & 226 & 27.9 & $\%$ & & 226 & 28.3 & $\%$ & & 228 & 27.6 & $\%$ & & 0.043 \\
\hline Treated by OHA (\%) & 226 & 72.6 & $\%$ & & 227 & 75.8 & $\%$ & & 226 & 72.1 & $\%$ & & 228 & 74.6 & $\%$ & & 0.858 \\
\hline $\begin{array}{l}\text { Treated by } \\
\text { antihypertensives (\%) }\end{array}$ & 226 & 58.4 & $\%$ & & 225 & 58.7 & $\%$ & & 224 & 56.3 & $\%$ & & 228 & 55.3 & $\%$ & & 0.420 \\
\hline $\begin{array}{l}\text { Treated by ACE inhibitor } \\
(\%)\end{array}$ & 226 & 22.1 & $\%$ & & 225 & 24.9 & $\%$ & & 224 & 19.2 & $\%$ & & 228 & 20.2 & $\%$ & & 0.347 \\
\hline Treated by ARB (\%) & 226 & 9.7 & $\%$ & & 225 & 8.0 & $\%$ & & 224 & 7.6 & $\%$ & & 228 & 11.4 & $\%$ & & 0.588 \\
\hline $\begin{array}{l}\text { Treated by calcium } \\
\text { channel blockers }(\%)\end{array}$ & 226 & 44.7 & $\%$ & & 225 & 44.0 & $\%$ & & 224 & 42.0 & $\%$ & & 228 & 40.4 & $\%$ & & 0.306 \\
\hline $\begin{array}{l}\text { Treated by lipid-lowering } \\
\text { agents }(\%)\end{array}$ & 226 & 42.0 & $\%$ & & 227 & 40.1 & $\%$ & & 225 & 41.8 & $\%$ & & 228 & 35.1 & $\%$ & & 0.186 \\
\hline $\begin{array}{l}\text { Treated by antiplatelet } \\
\text { agents }(\%)\end{array}$ & 227 & 29.1 & $\%$ & & 227 & 25.1 & $\%$ & & 224 & 31.7 & $\%$ & & 228 & 25.0 & $\%$ & & 0.667 \\
\hline $\begin{array}{l}\text { Treated by anticoagulants } \\
(\%) \\
\text { History of complications }\end{array}$ & 226 & 3.1 & $\%$ & & 226 & 2.7 & $\%$ & & 225 & 1.8 & $\%$ & & 226 & 1.8 & $\%$ & & 0.282 \\
\hline $\begin{array}{l}\text { Cardiovascular disease } \\
(\%)\end{array}$ & 228 & 28.1 & $\%$ & & 228 & 30.7 & $\%$ & & 228 & 33.8 & $\%$ & & 228 & 20.6 & $\%$ & & 0.148 \\
\hline Nephropathy (\%) & 209 & 18.2 & $\%$ & & 215 & 13.0 & $\%$ & & 213 & 15.5 & $\%$ & & 216 & 13.9 & $\%$ & & 0.350 \\
\hline Retinopathy (\%) & 228 & 44.7 & $\%$ & & 228 & 45.6 & $\%$ & & 228 & 42.1 & $\%$ & & 228 & 33.8 & $\%$ & & 0.013 \\
\hline $\begin{array}{l}\text { Leisure-time physical } \\
\text { activity (METs) }\end{array}$ & 198 & 12.1 & \pm & 18.9 & 189 & 15.1 & \pm & 27.9 & 195 & 15.2 & \pm & 28.5 & 189 & 15.4 & \pm & 26.9 & 0.214 \\
\hline Current smoker $(\%)$ & 213 & 14.6 & $\%$ & & 214 & 14.5 & $\%$ & & 214 & 14.0 & $\%$ & & 219 & 18.7 & $\%$ & & 0.270 \\
\hline $\begin{array}{l}\text { Current alcohol drinker } \\
(\%)\end{array}$ & 211 & 24.2 & $\%$ & & 214 & 29.9 & $\%$ & & 214 & 31.3 & $\%$ & & 219 & 30.1 & $\%$ & & 0.167 \\
\hline
\end{tabular}

Abbreviations: ACE, angiotensin-converting enzyme; ARB, angiotensin II receptor blocker; eGFR, estimated glomerular filtration rate; OHA, oral hypoglycemic agent. 
Table 2 shows baseline daily dietary intake by the study patients with type 2 diabetes according to quartiles of sodium intake. Increases in total energy intake were observed from the first to the fourth quartile groups of sodium intake (Q1, $1425.2 \mathrm{kcal} ; \mathrm{Q} 2,1647.3 \mathrm{kcal}$; Q3, $1796.1 \mathrm{kcal}$; and Q4, $2033.3 \mathrm{kcal} ; p<0.001)$. The proportions of fat and protein as percentages of the energy supply in the higher sodium intake quartiles $(\mathrm{Q} 2, \mathrm{Q} 3$, and Q4) were significantly increased compared with the lowest sodium intake quartile (Q1), while the proportion of carbohydrates as the percentage of the energy supply were significantly decreased in the Q2, Q3, and Q4 quartiles (all $p<0.001$ ). Patients with higher quartiles of sodium intake had significantly greater intakes of vitamins, minerals, and each food group.

Table 2. Baseline food groups and nutritional intakes of the 912 elderly patients with type 2 diabetes according to quartiles of sodium intake.

\begin{tabular}{|c|c|c|c|c|c|c|c|c|c|c|c|c|c|}
\hline & \multicolumn{3}{|c|}{$\mathrm{Q} 1(n=228)$} & \multicolumn{3}{|c|}{$\mathrm{Q} 2(n=228)$} & \multicolumn{3}{|c|}{ Q3 $(n=228)$} & \multicolumn{3}{|c|}{$\mathrm{Q} 4(n=228)$} & \multirow{2}{*}{$\begin{array}{c}\text { Trend } \\
p\end{array}$} \\
\hline & Mean & \pm & SD & Mean & \pm & SD & Mean & \pm & SD & Mean & \pm & SD & \\
\hline Sodium intake (g/day) & 2.5 & \pm & 0.5 & 3.5 & \pm & 0.2 & 4.4 & \pm & 0.3 & 5.9 & \pm & 0.9 & $<0.001$ \\
\hline \multicolumn{14}{|l|}{ Nutritional intake } \\
\hline Energy (kcal/day) & 1425.2 & \pm & 240.6 & 1647.3 & \pm & 261.3 & 1796.1 & \pm & 296.3 & 2033.3 & \pm & 379.2 & $<0.001$ \\
\hline Protein (g/day) & 52.7 & \pm & 13.2 & 63.2 & \pm & 13.2 & 70.7 & \pm & 15.7 & 81.2 & \pm & 19.6 & $<0.001$ \\
\hline Protein (\% energy) & 14.7 & \pm & 2.2 & 15.4 & \pm & 2.1 & 15.7 & \pm & 2.0 & 16.0 & \pm & 2.2 & $<0.001$ \\
\hline Fat (g/day) & 37.7 & \pm & 11.6 & 47.1 & \pm & 12.5 & 52.5 & \pm & 13.8 & 62.4 & \pm & 20.1 & $<0.001$ \\
\hline Fat ( $\%$ energy) & 23.5 & \pm & 4.7 & 25.6 & \pm & 4.4 & 26.1 & \pm & 4.1 & 27.2 & \pm & 4.9 & $<0.001$ \\
\hline Carbohydrate (g/day) & 210.7 & \pm & 30.6 & 233.1 & \pm & 37.5 & 250.2 & \pm & 38.5 & 276.7 & \pm & 47.6 & $<0.001$ \\
\hline Carbohydrate (\% energy) & 61.8 & \pm & 6.0 & 59.1 & \pm & 5.5 & 58.2 & \pm & 5.3 & 56.8 & \pm & 5.8 & $<0.001$ \\
\hline Fiber, total (g/day) & 10.9 & \pm & 3.2 & 13.4 & \pm & 3.6 & 14.9 & \pm & 3.7 & 17.2 & \pm & 4.2 & $<0.001$ \\
\hline Vitamin A ( $\mu$ g RAE/day) & 803.4 & \pm & 362.7 & 993.4 & \pm & 404.6 & 1055.6 & \pm & 415.1 & 1187.1 & \pm & 433.8 & $<0.001$ \\
\hline Vitamin D ( $\mu \mathrm{g} /$ day $)$ & 7.1 & \pm & 3.6 & 9.1 & \pm & 3.7 & 10.5 & \pm & 4.4 & 12.4 & \pm & 5.4 & $<0.001$ \\
\hline Vitamin E (mg/day) & 5.8 & \pm & 1.5 & 7.3 & \pm & 1.7 & 8.0 & \pm & 1.7 & 9.4 & \pm & 2.2 & $<0.001$ \\
\hline Vitamin K (mg/day) & 187.1 & \pm & 75.0 & 234.0 & \pm & 84.8 & 251.2 & \pm & 88.3 & 291.1 & \pm & 96.1 & $<0.001$ \\
\hline Vitamin B1 (mg/day) & 0.7 & \pm & 0.2 & 0.8 & \pm & 0.2 & 0.9 & \pm & 0.2 & 1.1 & \pm & 0.3 & $<0.001$ \\
\hline Vitamin B2 (mg/day) & 0.8 & \pm & 0.2 & 1.0 & \pm & 0.2 & 1.1 & \pm & 0.3 & 1.3 & \pm & 0.3 & $<0.001$ \\
\hline Niacin $(m$ & 11.5 & \pm & 3.8 & 14.1 & \pm & 3.9 & 16.0 & \pm & 4.5 & 18.6 & \pm & 5.7 & $<0.001$ \\
\hline Vitamin B6 (mg/day) & 1.0 & \pm & 0.3 & 1.2 & \pm & 0.3 & 1.3 & \pm & 0.3 & 1.5 & \pm & 0.4 & $<0.001$ \\
\hline Vitamin B12 ( $\mu \mathrm{g} /$ day $)$ & 6.0 & \pm & 2.9 & 7.5 & \pm & 3.0 & 8.8 & \pm & 3.7 & 10.4 & \pm & 4.5 & $<0.001$ \\
\hline Folate (mg/day) & 250.1 & \pm & 81.0 & 303.6 & \pm & 90.6 & 331.5 & \pm & 97.5 & 375.6 & \pm & 100.2 & $<0.001$ \\
\hline Pantothenic acid (mg/day) & 4.5 & \pm & 1.0 & 5.2 & \pm & 1.0 & 5.7 & \pm & 1.1 & 6.4 & \pm & 1.4 & $<0.001$ \\
\hline Vitamin C (mg/day) & 87.8 & \pm & 36.5 & 106.3 & \pm & 40.9 & 119.3 & \pm & 41.7 & 137.2 & \pm & 44.6 & $<0.001$ \\
\hline Potassium (mg/day) & 1926.8 & \pm & 515.0 & 2334.8 & \pm & 563.2 & 2636.5 & \pm & 585.1 & 3033.0 & \pm & 680.6 & $<0.001$ \\
\hline Calcium (mg/day) & 479.5 & \pm & 148.6 & 580.5 & \pm & 165.0 & 650.9 & \pm & 165.5 & 734.9 & \pm & 212.7 & $<0.001$ \\
\hline Magnes & 209.3 & \pm & 49.0 & 251.5 & \pm & 55.7 & 278.5 & \pm & 55.8 & 323.0 & \pm & 69.3 & $<0.001$ \\
\hline P (mg/day) & 837.5 & \pm & 202.0 & 993.3 & \pm & 206.3 & 1106.8 & \pm & 229.0 & 1262.2 & \pm & 285.1 & $<0.001$ \\
\hline $\mathrm{Fe}$ (mg/day) & 6.6 & \pm & 1.5 & 8.0 & \pm & 1.8 & 8.8 & \pm & 1.9 & 10.2 & \pm & 2.3 & $<0.001$ \\
\hline $\mathrm{Zn}(\mu \mathrm{g} /$ day $)$ & 16.1 & \pm & 12.5 & 24.0 & \pm & 18.4 & 31.8 & \pm & 24.8 & 42.2 & \pm & 33.0 & $<0.001$ \\
\hline $\mathrm{Cu}$ (mg/day) & 1.4 & \pm & 0.6 & 1.9 & \pm & 0.9 & 2.3 & \pm & 1.2 & 3.0 & \pm & 1.6 & $<0.001$ \\
\hline Mn (mg/day) & 2.4 & \pm & 0.5 & 2.7 & \pm & 0.6 & 2.8 & \pm & 0.5 & 3.2 & \pm & 0.6 & $<0.001$ \\
\hline \multicolumn{14}{|l|}{ Intake of food groups } \\
\hline Grain (g/day) & 192.3 & \pm & 29.1 & 196.7 & \pm & 3 & 197 & \pm & 3 & 20 & \pm & 40.1 & $<0.001$ \\
\hline s (g/day) & 30.7 & \pm & 28.3 & 34.8 & \pm & 24.8 & 47.4 & \pm & 32.9 & 50.3 & \pm & 33.1 & $<0.001$ \\
\hline Vegetables (g/day) & 206.519 & \pm & 107.6 & 258.824 & \pm & 116.6 & 285.662 & \pm & 132.1 & 323.675 & \pm & 128.7 & $<0.001$ \\
\hline Fruits (g/day) & 102.5 & \pm & 68.7 & 118.0 & \pm & 82.2 & 132.6 & \pm & 77.3 & 159.0 & \pm & 83.6 & $<0.001$ \\
\hline Seaweed (g/day) & 2.1 & \pm & 1.5 & 2.5 & \pm & 1.5 & 2.7 & \pm & 1.9 & 3.2 & \pm & 2.0 & $<0.001$ \\
\hline Meat (g/day) & 30.7 & \pm & 27.8 & 41.4 & \pm & 33.1 & 46.5 & \pm & 35.9 & 58.0 & \pm & 56.6 & $<0.001$ \\
\hline Fish/Seafood (g/day) & 62.6 & \pm & 38.4 & 78.8 & \pm & 41.3 & 94.6 & \pm & 50.1 & 112.0 & \pm & 60.0 & $<0.001$ \\
\hline Eggs (g/day) & 23.5 & \pm & 15.8 & 26.6 & \pm & 15.8 & 28.0 & \pm & 17.6 & 31.9 & \pm & 20.3 & $<0.001$ \\
\hline $\begin{array}{l}\text { Soybeans/Soy products } \\
\text { (g/day) }\end{array}$ & 55.7 & \pm & 3 & 6 & \pm & 35 & 9 & \pm & 41.6 & 3 & \pm & 47.8 & $<0.001$ \\
\hline Milk/Dairy products (g/day) & 140.8 & \pm & 80.3 & 151.5 & \pm & 89.1 & 171.5 & \pm & 94.0 & 175.9 & \pm & 126.8 & $<0.001$ \\
\hline Snacks/Candies (g/day) & 26.8 & \pm & 24.2 & 39.8 & \pm & 32.1 & 47.6 & \pm & 38.9 & 58.3 & \pm & 49.3 & $<0.001$ \\
\hline Ethanol (g/day) & 3.0 & \pm & 11.8 & 3.8 & \pm & 16.0 & 3.9 & \pm & 12.3 & 6.3 & \pm & 22.3 & 0.041 \\
\hline
\end{tabular}


During the six-year follow-up, in those in the first to the fourth quartiles of sodium intake there were 28, 17, 29, and 42 incidents of diabetic retinopathy, 1, 5, 7, and 4 incidents of overt nephropathy, and 17, 12, 11, and 12 incidents of CVD, respectively. Deaths according to the first to the fourth quartiles of sodium intake were 28, 19, 16, and 19, respectively. Table 3 shows HRs for sodium intake estimated by Cox regression models unadjusted (top model), adjusted for risk factors (middle model), and further adjusted for the use of ARB, ACE inhibitors, and calcium channel blockers at baseline (bottom model). In confounder-adjusted Cox regression, HRs and $95 \%$ CI for diabetic complications in patients in the second, third, and fourth quartiles of sodium intake were calculated using those in the first quartile of sodium intake as the reference. There were no significant associations between sodium intake quartiles and incidence of diabetes complications and mortality under the fully adjusted model (diabetic retinopathy: 0.92 (95\% CI, 0.46-1.84), $p=0.802,1.52(0.79-2.92), p=0.214$, and $1.72(0.91-3.24), p=0.096$; overt nephropathy: 3.33 (0.35-31.76), $p=0.296,3.95(0.40-38.93), p=0.24$, and $2.16(0.17-27.12), p=0.552$; CVD: 0.92 (0.38-2.22), $p=0.846,0.76(0.30-1.94), p=0.573$, and $1.21(0.46-3.21), p=0.703)$ and mortality: 0.80 (0.38-1.66), $p=0.54,0.80(0.37-1.73), p=0.565$, and $1.03(0.44-2.44)$, $p=0.948)$. However, a significant positive linear trend was shown between sodium intake and the risk of diabetic retinopathy (trend $p=0.039$ ). There was no significant linear trend between sodium intake and the risk of overt nephropathy, CVD, and mortality (trend $p=0.729,0.905$, and 0.460 , respectively).

Table 3. Cox regression analysis of diabetes complications and all-cause mortality according to quartiles of sodium intake.

\begin{tabular}{|c|c|c|c|c|c|c|c|c|c|c|c|c|c|c|c|}
\hline & \multicolumn{2}{|l|}{ Q1 } & \multicolumn{4}{|c|}{ Q2 } & \multicolumn{4}{|c|}{ Q3 } & \multicolumn{4}{|c|}{ Q4 } & \multirow{2}{*}{$\begin{array}{c}\text { Trend } \\
\quad p\end{array}$} \\
\hline & Events/Pts & HR & Events/Pts & HR & $95 \% \mathrm{CI}$ & $p$ & Events/Pts & HR & $95 \% \mathrm{CI}$ & $p$ & Events/Pts & HR & $95 \% \mathrm{CI}$ & $p$ & \\
\hline \multicolumn{16}{|c|}{ Diabetic retinopathy $(n=327)$} \\
\hline Not adjusted & $28 / 82$ & ref & $17 / 65$ & 0.72 & $\begin{array}{l}0.39- \\
1.31\end{array}$ & 0.276 & $29 / 75$ & 0.94 & $\begin{array}{l}0.56- \\
1.58\end{array}$ & 0.820 & $42 / 105$ & 1.04 & $\begin{array}{l}0.65- \\
1.69\end{array}$ & 0.861 & 0.202 \\
\hline Adjusted * & $21 / 68$ & ref & $14 / 53$ & 0.96 & $\begin{array}{l}0.48- \\
1.92\end{array}$ & 0.904 & $24 / 63$ & 1.32 & $\begin{array}{l}0.69- \\
2.49\end{array}$ & 0.401 & $36 / 83$ & 1.66 & $\begin{array}{l}0.88- \\
3.13\end{array}$ & 0.115 & 0.059 \\
\hline Further Adjusted ** & $21 / 68$ & ref & $14 / 53$ & 0.92 & $\begin{array}{l}0.46- \\
1.84\end{array}$ & 0.802 & $24 / 62$ & 1.52 & $\begin{array}{l}0.79- \\
2.92\end{array}$ & 0.214 & $36 / 83$ & 1.72 & $\begin{array}{l}0.91- \\
3.24\end{array}$ & 0.096 & 0.039 \\
\hline \multicolumn{16}{|c|}{ Diabetic overt nephropathy $(n=624)$} \\
\hline Not adjusted & $1 / 145$ & ref & $5 / 169$ & 3.62 & $\begin{array}{l}0.40- \\
32.34\end{array}$ & 0.250 & $7 / 158$ & 6.36 & $\begin{array}{l}0.78- \\
51.67\end{array}$ & 0.084 & $4 / 152$ & 3.90 & $\begin{array}{l}0.44- \\
34.85\end{array}$ & 0.224 & 0.233 \\
\hline Adjusted * & $1 / 118$ & ref & $4 / 133$ & 3.97 & $\begin{array}{l}0.41- \\
38.23\end{array}$ & 0.233 & $7 / 133$ & 4.80 & $\begin{array}{l}0.50- \\
46.04\end{array}$ & 0.174 & $4 / 121$ & 2.37 & $\begin{array}{l}0.19- \\
30.01\end{array}$ & 0.505 & 0.671 \\
\hline Further Adjusted ** & $1 / 118$ & ref & $4 / 133$ & 3.33 & $\begin{array}{l}0.35- \\
31.76\end{array}$ & 0.296 & $7 / 133$ & 3.95 & $\begin{array}{l}0.40- \\
38.93\end{array}$ & 0.240 & $4 / 121$ & 2.16 & $\begin{array}{l}0.17- \\
27.12\end{array}$ & 0.552 & 0.729 \\
\hline \multicolumn{16}{|c|}{ Cardiovascular disease $(n=651)$} \\
\hline Not adjusted & $17 / 162$ & ref & $12 / 157$ & 0.74 & $\begin{array}{l}0.35- \\
1.55\end{array}$ & 0.423 & $11 / 151$ & 0.66 & $\begin{array}{l}0.31- \\
1.40\end{array}$ & 0.277 & $12 / 181$ & 0.62 & $\begin{array}{l}0.30- \\
1.31\end{array}$ & 0.210 & 0.205 \\
\hline Adjusted * & $14 / 137$ & ref & $9 / 119$ & 0.94 & $\begin{array}{l}0.39- \\
2.28\end{array}$ & 0.892 & $10 / 132$ & 0.85 & $\begin{array}{l}0.34- \\
2.08\end{array}$ & 0.715 & $11 / 147$ & 1.20 & $\begin{array}{l}0.45- \\
3.19\end{array}$ & 0.719 & 0.876 \\
\hline Further Adjusted ** & $14 / 136$ & ref & $9 / 118$ & 0.92 & $\begin{array}{l}0.38- \\
2.22\end{array}$ & 0.846 & $9 / 130$ & 0.76 & $\begin{array}{l}0.30- \\
1.94\end{array}$ & 0.573 & $11 / 147$ & 1.21 & $\begin{array}{l}0.46- \\
3.21\end{array}$ & 0.703 & 0.905 \\
\hline \multicolumn{16}{|c|}{ All-cause mortality $(n=912)$} \\
\hline Not adjusted & $28 / 228$ & ref & $15 / 228$ & 0.63 & $\begin{array}{l}0.33- \\
1.17\end{array}$ & 0.143 & $19 / 228$ & 0.76 & $\begin{array}{l}0.42- \\
1.37\end{array}$ & 0.368 & $16 / 228$ & 0.62 & $\begin{array}{l}0.33- \\
1.15\end{array}$ & 0.131 & 0.092 \\
\hline Adjusted * & 23/191 & ref & $13 / 178$ & 0.79 & $\begin{array}{l}0.38- \\
1.64\end{array}$ & 0.530 & 14/191 & 0.94 & $\begin{array}{l}0.45- \\
1.97\end{array}$ & 0.865 & $13 / 184$ & 1.02 & $\begin{array}{l}0.43- \\
2.40\end{array}$ & 0.964 & 0.510 \\
\hline Further Adjusted ** & $23 / 190$ & ref & $13 / 177$ & 0.80 & $\begin{array}{l}0.38- \\
1.66\end{array}$ & 0.540 & $12 / 189$ & 0.80 & $\begin{array}{l}0.37- \\
1.73\end{array}$ & 0.565 & $13 / 184$ & 1.03 & $\begin{array}{l}0.44- \\
2.44\end{array}$ & 0.948 & 0.460 \\
\hline
\end{tabular}

* Adjusted for age, sex, BMI, HbA1c, diabetes duration, LDL-cholesterol, HDL-cholesterol, log-transformed triglycerides, treatment by insulin, current smoker, alcohol intake, energy intake, physical activity, and systolic blood pressure. ${ }^{* *}$ Further adjusted for the use of ARB, ACE inhibitors, and calcium channel blockers at baseline.

The results of subgroup analysis of quartiles of sodium consumption and the incidence of diabetic retinopathy according to gender, age, duration of diabetes, $\mathrm{HbA1c}$, SBP, protein intake, and vegetable intake are shown in Table 4. Subgroup analyses according to gender (men vs. women), age ( $<75$ years, $\geq 75$ years), duration of diabetes (whether $<$ mean value (16.4 years) or not), HbA1c ( $<8.5 \%$ vs. $\geq 8.5 \%)$, SBP ( $<140 \mathrm{mmHg}$ vs. $\geq 140 \mathrm{mmHg})$, and protein intake (whether $<$ mean value $(66.9 \mathrm{~g})$ or not) did not reveal significant associations between quartiles of sodium intake and incident diabetic retinopathy. Among patients 
whose vegetable intake was $<268.7 \mathrm{~g} /$ day, patients in the highest and second-highest quartiles (Q4 and Q3) of sodium intake had a significantly higher incidence of diabetic retinopathy compared with the first quartile (Q2: $0.87(0.31-2.41), p=0.785$; Q3: $2.61(1.00$ 6.83), $p=0.050$; and Q4: 3.70 (1.37-10.02), $p=0.010$ ). However, there was no significant differences between quartiles of sodium intake when the analysis was restricted to patients whose vegetable intake was $\geq 268.7 \mathrm{~g} /$ day (equal to or higher than participants' mean vegetable intake) $(\mathrm{Q} 2: 1.57(0.45-5.44), p=0.478$; $\mathrm{Q} 3: 1.91$ (0.56-6.55), $p=0.306$; and Q4: 1.51 $(0.48-4.72), p=0.483)$. As for a linear trend, a significant positive trend was shown between sodium intake and the risk of diabetic retinopathy in patients younger than 75 years, whose diabetes duration was lower than the average of 16.4 years, or whose vegetable intake was less than $268.7 \mathrm{~g} /$ day (trend $p=0.025,0.028,0.049$, respectively). There was no significant linear trend between sodium intake and the risk of diabetic retinopathy in other patient groups.

Table 4. Subgroup analysis of quartile of sodium and incidence of diabetic retinopathy.

\begin{tabular}{|c|c|c|c|c|c|c|c|c|c|c|c|c|c|c|c|}
\hline & \multicolumn{2}{|c|}{$\mathrm{Q} 1(n=82)$} & \multicolumn{4}{|c|}{$\mathrm{Q} 2(n=65)$} & \multicolumn{3}{|c|}{ Q3 $(n=75)$} & \multicolumn{4}{|c|}{$\mathrm{Q} 4(n=105)$} & \multirow[b]{2}{*}{$p$} & \multirow{2}{*}{$\begin{array}{c}\text { Trend } \\
\quad p\end{array}$} \\
\hline & Events/Pts & HR & Events/Pts & HR & $95 \% \mathrm{CI}$ & $p$ & Events/Pts & HR & $95 \% \mathrm{CI}$ & $p$ & Events/Pts & HR & $95 \% \mathrm{CI}$ & & \\
\hline \multicolumn{16}{|l|}{ Age $<75$ y $(n=237)$} \\
\hline Not adjusted & $19 / 62$ & ref & $14 / 42$ & 1.06 & $\begin{array}{l}0.51- \\
2.18\end{array}$ & 0.880 & $24 / 56$ & 1.30 & $\begin{array}{l}0.70- \\
2.42\end{array}$ & 0.413 & $31 / 77$ & 1.41 & $\begin{array}{l}0.78- \\
2.55\end{array}$ & 0.262 & 0.173 \\
\hline Adjusted * & $15 / 53$ & ref & $12 / 36$ & 1.33 & $\begin{array}{l}0.60- \\
2.93\end{array}$ & 0.484 & $20 / 46$ & 1.75 & $\begin{array}{l}0.84- \\
3.63\end{array}$ & 0.136 & $26 / 61$ & 1.97 & $\begin{array}{l}0.93- \\
4.19\end{array}$ & 0.077 & 0.048 \\
\hline Further Adjusted ** & $15 / 53$ & ref & $12 / 36$ & 1.24 & $\begin{array}{l}0.56- \\
2.73\end{array}$ & 0.602 & $20 / 46$ & 2.13 & $\begin{array}{l}0.99- \\
4.56\end{array}$ & 0.052 & $26 / 61$ & 2.12 & $\begin{array}{l}1.00- \\
4.50\end{array}$ & 0.050 & 0.025 \\
\hline \multicolumn{16}{|l|}{ Age $\geq 75$ y $(n=90)$} \\
\hline Not adjusted & $9 / 20$ & ref & $3 / 23$ & 0.30 & $\begin{array}{l}0.08- \\
1.14\end{array}$ & 0.077 & $5 / 19$ & 0.32 & $\begin{array}{l}0.09- \\
1.11\end{array}$ & 0.072 & $11 / 28$ & 0.72 & $\begin{array}{l}0.29- \\
1.81\end{array}$ & 0.490 & 0.859 \\
\hline Adjusted * & $6 / 15$ & ref & $2 / 17$ & 0.06 & $\begin{array}{l}0.01- \\
0.70\end{array}$ & 0.024 & $4 / 17$ & 0.13 & $\begin{array}{l}0.02- \\
1.11\end{array}$ & 0.062 & $10 / 22$ & 0.29 & $\begin{array}{l}0.03- \\
2.46\end{array}$ & 0.255 & 0.436 \\
\hline Further Adjusted $* *$ & $6 / 15$ & ref & $2 / 17$ & 0.08 & $\begin{array}{l}0.01- \\
0.84\end{array}$ & 0.035 & $4 / 16$ & 0.20 & $\begin{array}{l}0.02- \\
2.10\end{array}$ & 0.180 & $10 / 22$ & 0.48 & $\begin{array}{l}0.04- \\
5.79\end{array}$ & 0.564 & 0.426 \\
\hline \multicolumn{16}{|l|}{ Males $(n=156)$} \\
\hline Not adjusted & $12 / 38$ & ref & $9 / 27$ & 1.03 & $\begin{array}{l}0.43- \\
2.50\end{array}$ & 0.948 & $18 / 38$ & 1.15 & $\begin{array}{l}0.54- \\
2.47\end{array}$ & 0.714 & $17 / 53$ & 0.89 & $\begin{array}{l}0.42- \\
1.92\end{array}$ & 0.772 & 0.782 \\
\hline Adjusted * & $10 / 32$ & ref & $7 / 22$ & 1.19 & $\begin{array}{l}0.42- \\
3.37\end{array}$ & 0.742 & $15 / 31$ & 1.76 & $\begin{array}{l}0.73- \\
4.27\end{array}$ & 0.211 & $14 / 43$ & 1.32 & $\begin{array}{l}0.53- \\
3.30\end{array}$ & 0.557 & 0.285 \\
\hline Further Adjusted ** & $10 / 32$ & ref & $7 / 22$ & 1.10 & $\begin{array}{l}0.37- \\
3.24\end{array}$ & 0.864 & $15 / 31$ & 1.84 & $\begin{array}{l}0.74- \\
4.53\end{array}$ & 0.187 & $14 / 43$ & 1.36 & $\begin{array}{l}0.53- \\
3.45\end{array}$ & 0.521 & 0.214 \\
\hline \multicolumn{16}{|l|}{ Females $(n=171)$} \\
\hline Not adjusted & $16 / 44$ & ref & $8 / 38$ & 0.59 & $\begin{array}{l}0.24- \\
1.45\end{array}$ & 0.248 & $11 / 37$ & 0.80 & $\begin{array}{l}0.36- \\
1.76\end{array}$ & 0.572 & $25 / 52$ & 1.53 & $\begin{array}{l}0.79- \\
2.94\end{array}$ & 0.205 & 0.135 \\
\hline Adjusted * & $11 / 36$ & ref & $7 / 31$ & 0.67 & $\begin{array}{l}0.24- \\
1.93\end{array}$ & 0.462 & $9 / 32$ & 0.84 & $\begin{array}{l}0.30- \\
2.39\end{array}$ & 0.745 & $22 / 40$ & 1.58 & $\begin{array}{l}0.60- \\
4.15\end{array}$ & 0.353 & 0.253 \\
\hline Further Adjusted ** & $11 / 36$ & ref & $7 / 31$ & 0.66 & $\begin{array}{l}0.23- \\
1.91 \\
\end{array}$ & 0.441 & $9 / 31$ & 0.91 & $\begin{array}{l}0.30- \\
2.76\end{array}$ & 0.861 & $22 / 40$ & 1.68 & $\begin{array}{l}0.61- \\
4.63 \\
\end{array}$ & 0.315 & 0.276 \\
\hline \multicolumn{16}{|c|}{ Duration of diabetes $<16.4$ y $(n=221)$} \\
\hline Not adjusted & $19 / 56$ & ref & $11 / 42$ & 0.73 & $\begin{array}{l}0.34- \\
1.57\end{array}$ & 0.420 & $19 / 55$ & 0.87 & $\begin{array}{l}0.44- \\
1.68\end{array}$ & 0.668 & $29 / 68$ & 1.28 & $\begin{array}{l}0.69- \\
2.35\end{array}$ & 0.434 & 0.212 \\
\hline Adjusted * & $12 / 46$ & ref & $9 / 38$ & 0.93 & $\begin{array}{l}0.37- \\
2.30\end{array}$ & 0.869 & $16 / 47$ & 1.09 & $\begin{array}{l}0.49- \\
2.41\end{array}$ & 0.833 & $26 / 57$ & 1.78 & $\begin{array}{l}0.81- \\
3.93\end{array}$ & 0.152 & 0.037 \\
\hline Further Adjusted ** & $12 / 46$ & ref & $9 / 38$ & 0.94 & $\begin{array}{l}0.38- \\
2.36\end{array}$ & 0.897 & $16 / 47$ & 1.15 & $\begin{array}{l}0.51- \\
2.59\end{array}$ & 0.745 & $26 / 57$ & 1.85 & $\begin{array}{l}0.83- \\
4.12\end{array}$ & 0.131 & 0.028 \\
\hline \multicolumn{16}{|c|}{ Duration of diabetes $\geq 16.4$ y $(n=101)$} \\
\hline Not adjusted & $9 / 26$ & ref & $6 / 20$ & 1.23 & $\begin{array}{l}0.40- \\
3.76\end{array}$ & 0.719 & $10 / 19$ & 1.56 & $\begin{array}{l}0.60- \\
4.05\end{array}$ & 0.363 & $13 / 36$ & 1.10 & $\begin{array}{l}0.46- \\
2.66\end{array}$ & 0.833 & 0.665 \\
\hline Adjusted * & $9 / 22$ & ref & $5 / 15$ & 1.95 & $\begin{array}{l}0.47- \\
8.11\end{array}$ & 0.360 & $8 / 16$ & 4.26 & $\begin{array}{l}1.00- \\
18.16\end{array}$ & 0.050 & $10 / 26$ & 2.69 & $\begin{array}{l}0.72- \\
10.01\end{array}$ & 0.140 & 0.534 \\
\hline Further Adjusted ** & $9 / 22$ & ref & $5 / 15$ & 2.32 & $\begin{array}{c}0.49- \\
10.88 \\
\end{array}$ & 0.287 & $8 / 15$ & 5.79 & $\begin{array}{l}1.12- \\
30.11 \\
\end{array}$ & 0.037 & $10 / 26$ & 2.95 & $\begin{array}{c}0.80- \\
10.97\end{array}$ & 0.106 & 0.404 \\
\hline \multicolumn{16}{|l|}{$\mathrm{HbA} 1 \mathrm{c}<8.5 \%(n=213)$} \\
\hline Not adjusted & $15 / 52$ & ref & $9 / 42$ & 0.88 & $\begin{array}{l}0.36- \\
2.17\end{array}$ & 0.783 & $17 / 48$ & 1.05 & $\begin{array}{l}0.50- \\
2.22\end{array}$ & 0.898 & $28 / 71$ & 1.61 & $\begin{array}{l}0.81- \\
3.18\end{array}$ & 0.174 & 0.099 \\
\hline Adjusted * & $12 / 43$ & ref & $7 / 33$ & 0.98 & $\begin{array}{l}0.37- \\
2.61\end{array}$ & 0.967 & $12 / 38$ & 1.10 & $\begin{array}{l}0.47- \\
2.58\end{array}$ & 0.835 & $24 / 55$ & 2.20 & $\begin{array}{l}0.94- \\
5.16\end{array}$ & 0.070 & 0.075 \\
\hline Further Adjusted ** & $12 / 43$ & ref & $7 / 33$ & 0.95 & $\begin{array}{l}0.36- \\
2.54\end{array}$ & 0.922 & $12 / 37$ & 1.14 & $\begin{array}{l}0.47- \\
2.78\end{array}$ & 0.768 & $24 / 55$ & 2.22 & $\begin{array}{l}0.93- \\
5.29\end{array}$ & 0.073 & 0.074 \\
\hline \multicolumn{16}{|c|}{$\mathrm{HbA} 1 \mathrm{c} \geq 8.5 \%(n=114)$} \\
\hline Not adjusted & $13 / 30$ & ref & $8 / 23$ & 0.66 & $\begin{array}{l}0.27- \\
1.59\end{array}$ & 0.351 & $12 / 27$ & 1.06 & $\begin{array}{l}0.48- \\
2.33\end{array}$ & 0.894 & $14 / 34$ & 0.83 & $\begin{array}{l}0.39- \\
1.77\end{array}$ & 0.633 & 0.974 \\
\hline Adjusted * & $9 / 25$ & ref & $7 / 20$ & 0.77 & $\begin{array}{l}0.27- \\
2.18\end{array}$ & 0.619 & $12 / 25$ & 0.76 & $\begin{array}{l}0.22- \\
2.63\end{array}$ & 0.666 & $12 / 28$ & 0.69 & $\begin{array}{l}0.22- \\
2.21\end{array}$ & 0.535 & 0.681 \\
\hline Further Adjusted ** & $9 / 25$ & ref & $7 / 20$ & 0.59 & $\begin{array}{l}0.19- \\
1.82\end{array}$ & 0.361 & $12 / 25$ & 0.83 & $\begin{array}{c}0.25- \\
2.82\end{array}$ & 0.769 & $12 / 28$ & 0.51 & $\begin{array}{c}0.15- \\
1.79\end{array}$ & 0.295 & 0.683 \\
\hline
\end{tabular}


Table 4. Cont.

\begin{tabular}{|c|c|c|c|c|c|c|c|c|c|c|c|c|c|c|c|}
\hline & \multicolumn{2}{|c|}{ Q1 $(n=82)$} & \multicolumn{4}{|c|}{ Q2 $(n=65)$} & \multicolumn{3}{|c|}{ Q3 $(n=75)$} & \multicolumn{4}{|c|}{$\mathrm{Q} 4(n=105)$} & \multirow[b]{2}{*}{$p$} & \multirow{2}{*}{$\begin{array}{c}\text { Trend } \\
\qquad p\end{array}$} \\
\hline & Events/Pts & HR & Events/Pts & HR & $95 \% \mathrm{CI}$ & $p$ & Events/Pts & HR & $95 \% \mathrm{CI}$ & $p$ & Events/Pts & HR & $95 \% \mathrm{CI}$ & & \\
\hline \multicolumn{16}{|c|}{ Systolic blood pressure $<140 \mathrm{mmHg}(n=195)$} \\
\hline Not adjusted & $18 / 49$ & ref & $12 / 38$ & 0.93 & $\begin{array}{l}0.43- \\
2.01\end{array}$ & 0.850 & $14 / 44$ & 0.70 & $\begin{array}{l}0.34- \\
1.45\end{array}$ & 0.335 & $28 / 64$ & 1.31 & $\begin{array}{l}0.71- \\
2.44\end{array}$ & 0.390 & 0.408 \\
\hline Adjusted * & $14 / 40$ & ref & $10 / 31$ & 1.12 & $\begin{array}{l}0.48- \\
2.64\end{array}$ & 0.792 & $11 / 36$ & 0.98 & $\begin{array}{l}0.41- \\
2.37\end{array}$ & 0.966 & $23 / 51$ & 1.76 & $\begin{array}{c}0.74- \\
4.20\end{array}$ & 0.202 & 0.319 \\
\hline Further Adjusted ** & $14 / 40$ & ref & $10 / 31$ & 1.07 & $\begin{array}{l}0.45- \\
2.53\end{array}$ & 0.885 & $11 / 36$ & 1.17 & $\begin{array}{l}0.47- \\
2.89\end{array}$ & 0.734 & $23 / 51$ & 1.93 & $\begin{array}{l}0.81- \\
4.65\end{array}$ & 0.140 & 0.221 \\
\hline \multicolumn{16}{|c|}{ Systolic blood pressure $\geq 140 \mathrm{mmHg}(n=132)$} \\
\hline Not adjusted & $10 / 33$ & ref & $5 / 27$ & 0.60 & $\begin{array}{l}0.20- \\
1.79\end{array}$ & 0.360 & $15 / 31$ & 1.55 & $\begin{array}{l}0.67- \\
3.56\end{array}$ & 0.302 & $14 / 41$ & 1.07 & $\begin{array}{l}0.47- \\
2.48\end{array}$ & 0.868 & 0.317 \\
\hline Adjusted * & $7 / 28$ & ref & $4 / 22$ & 0.76 & $\begin{array}{l}0.21- \\
2.76\end{array}$ & 0.681 & $13 / 27$ & 2.22 & $\begin{array}{l}0.78- \\
6.34\end{array}$ & 0.136 & $13 / 32$ & 1.46 & $\begin{array}{l}0.46- \\
4.60\end{array}$ & 0.521 & 0.193 \\
\hline Further Adjusted ${ }^{* *}$ & $7 / 28$ & ref & $4 / 22$ & 0.74 & $\begin{array}{l}0.20- \\
2.70\end{array}$ & 0.646 & $13 / 26$ & 2.81 & $\begin{array}{l}0.88- \\
9.01\end{array}$ & 0.083 & $13 / 32$ & 1.84 & $\begin{array}{l}0.54- \\
6.33\end{array}$ & 0.333 & 0.230 \\
\hline \multicolumn{16}{|c|}{ Protein intake $<66.9 \mathrm{~g} /$ day $(n=136)$} \\
\hline Not adjusted & $22 / 64$ & ref & $12 / 42$ & 0.77 & $\begin{array}{l}0.37- \\
1.61\end{array}$ & 0.488 & $14 / 37$ & 1.05 & $\begin{array}{l}0.53- \\
2.08\end{array}$ & 0.891 & $8 / 23$ & 0.95 & $\begin{array}{l}0.42- \\
2.17\end{array}$ & 0.911 & 0.808 \\
\hline Adjusted * & $16 / 52$ & ref & $9 / 34$ & 0.91 & $\begin{array}{l}0.37- \\
2.19\end{array}$ & 0.825 & $12 / 30$ & 1.37 & $\begin{array}{l}0.57- \\
3.30\end{array}$ & 0.477 & $7 / 17$ & 0.93 & $\begin{array}{l}0.35- \\
2.47\end{array}$ & 0.877 & 0.426 \\
\hline Further Adjusted ** & $16 / 52$ & ref & $9 / 34$ & 0.83 & $\begin{array}{l}0.34- \\
2.04\end{array}$ & 0.683 & $12 / 30$ & 1.81 & $\begin{array}{l}0.69- \\
4.75\end{array}$ & 0.227 & $7 / 17$ & 1.32 & $\begin{array}{l}0.45- \\
3.87\end{array}$ & 0.611 & 0.238 \\
\hline \multicolumn{16}{|c|}{ Protein intake $\geq 66.9 \mathrm{~g} /$ day $(n=161)$} \\
\hline Not adjusted & $6 / 18$ & ref & $5 / 23$ & 0.91 & $\begin{array}{l}0.24- \\
3.39\end{array}$ & 0.886 & $15 / 38$ & 1.13 & $\begin{array}{l}0.37- \\
3.42\end{array}$ & 0.834 & $34 / 82$ & 1.48 & $\begin{array}{l}0.53- \\
4.19\end{array}$ & 0.456 & 0.188 \\
\hline Adjusted * & $5 / 16$ & ref & $5 / 19$ & 0.73 & $\begin{array}{l}0.18- \\
2.86\end{array}$ & 0.646 & $12 / 33$ & 0.96 & $\begin{array}{l}0.30- \\
3.11\end{array}$ & 0.949 & $29 / 66$ & 1.47 & $\begin{array}{l}0.48- \\
4.53\end{array}$ & 0.500 & 0.190 \\
\hline Further Adjusted ** & $5 / 16$ & ref & $5 / 19$ & 0.62 & $\begin{array}{l}0.15- \\
2.49\end{array}$ & 0.498 & $12 / 32$ & 1.01 & $\begin{array}{l}0.31- \\
3.31\end{array}$ & 0.983 & $29 / 66$ & 1.56 & $\begin{array}{l}0.52- \\
4.74\end{array}$ & 0.431 & 0.137 \\
\hline \multicolumn{16}{|c|}{ Vegetable intake $<268.7 \mathrm{~g} /$ day $(n=188)$} \\
\hline Not adjusted & $21 / 65$ & ref & $7 / 37$ & 0.61 & $\begin{array}{l}0.26- \\
1.44\end{array}$ & 0.258 & $16 / 47$ & 0.90 & $\begin{array}{l}0.46- \\
1.76\end{array}$ & 0.765 & $15 / 39$ & 1.18 & $\begin{array}{l}0.60- \\
2.32\end{array}$ & 0.638 & 0.400 \\
\hline Adjusted * & $16 / 53$ & ref & $6 / 32$ & 0.96 & $\begin{array}{l}0.35- \\
2.64\end{array}$ & 0.934 & $14 / 41$ & 1.81 & $\begin{array}{l}0.75- \\
4.34\end{array}$ & 0.185 & $14 / 31$ & 2.68 & $\begin{array}{l}1.07- \\
6.70\end{array}$ & 0.035 & 0.075 \\
\hline Further Adjusted ${ }^{* *}$ & $16 / 53$ & ref & $6 / 32$ & 0.87 & $\begin{array}{l}0.31- \\
2.41\end{array}$ & 0.785 & $14 / 40$ & 2.61 & $\begin{array}{l}1.00- \\
6.83\end{array}$ & 0.050 & $14 / 31$ & 3.70 & $\begin{array}{l}1.37- \\
10.02\end{array}$ & 0.010 & 0.049 \\
\hline \multicolumn{16}{|c|}{ Vegetable intake $\geq 268.7 \mathrm{~g} /$ day $(n=139)$} \\
\hline Not adjusted & $7 / 17$ & ref & $10 / 28$ & 1.14 & $\begin{array}{l}0.38- \\
3.39\end{array}$ & 0.819 & $13 / 28$ & 1.46 & $\begin{array}{l}0.51- \\
4.15\end{array}$ & 0.479 & $27 / 66$ & 1.42 & $\begin{array}{l}0.55- \\
3.70\end{array}$ & 0.471 & 0.828 \\
\hline Adjusted * & $5 / 15$ & ref & $8 / 21$ & 1.40 & $\begin{array}{l}0.42- \\
4.73\end{array}$ & 0.585 & $10 / 22$ & 1.44 & $\begin{array}{l}0.44- \\
4.68\end{array}$ & 0.547 & $22 / 52$ & 1.36 & $\begin{array}{l}0.44- \\
4.21\end{array}$ & 0.591 & 0.479 \\
\hline Further Adjusted ** & $5 / 15$ & ref & $8 / 21$ & 1.57 & $\begin{array}{l}0.45- \\
5.44\end{array}$ & 0.478 & $10 / 22$ & 1.91 & $\begin{array}{l}0.56- \\
6.55\end{array}$ & 0.306 & $22 / 52$ & 1.51 & $\begin{array}{l}0.48- \\
4.72\end{array}$ & 0.483 & 0.437 \\
\hline
\end{tabular}

* Adjusted for age, sex, BMI, HbA1c, diabetes duration, LDL-cholesterol, HDL-cholesterol, log-transformed triglycerides, treatment by insulin, current smoker, alcohol intake, energy intake, physical activity, and systolic blood pressure. ${ }^{* *}$ Further adjusted for the use of ARB, ACE inhibitors, and calcium channel blockers at baseline. In each subgroup analysis, the variable was not adjusted for.

\section{Discussion}

Guidelines for diabetes care [1-3] recommend a reduction in dietary sodium intake; however, there is no detailed evidence regarding the relationship between dietary sodium intake and the incidence of diabetes complications in elderly patients with type 2 diabetes. This six-year follow-up study of elderly Japanese patients with type 2 diabetes showed no significant associations of sodium intake with diabetic retinopathy, overt nephropathy, CVD, and all-cause mortality. However, high sodium intake under conditions of low vegetable intake was associated with an elevated incidence of diabetic retinopathy.

Our previous study based on data from the JDCS, which was mainly targeted toward middle-aged patients with type 2 diabetes, showed a significant relationship between high sodium intake and the incidence of CVD, although no such significant associations of high sodium intake with other diabetes complications and mortality were shown [6]. In that study, mean intakes of sodium by JDCS patients were Q1, 2.8 g/day; Q2, 3.8 g/day; Q3, $4.5 \mathrm{~g} /$ day; and Q4, $5.9 \mathrm{~g} /$ day. Those values were comparable to sodium intake by quartiles in the current J-EDIT study (Q1, 2.5 g/day; Q2, 3.5 g/day; Q3, $4.4 \mathrm{~g} /$ day; and Q4, $5.9 \mathrm{~g} /$ day). Current goals of daily dietary sodium intake for diabetes care are below $2.3 \mathrm{~g} /$ day in the guidelines of the American Diabetes Association (ADA) [1], $6 \mathrm{~g} /$ day according to the European Association for the Study of Diabetes (EASD) guidelines [2], and JDS recommends $7.5 \mathrm{~g} /$ day and $6.5 \mathrm{~g} /$ day salt intake for men and women, respectively, and $6 \mathrm{~g} /$ day for those with hypertension [3]. Comparing these guidelines with the lowest 
quartile of sodium consumption by J-EDIT patients, the J-EDIT patients consumed more sodium compared with that recommended by ADA and EASD guidelines and JDS guidelines for hypertensive patients with diabetes; however, intake was lower compared with JDS among normotensive men and women. The J-EDIT patients $(3.8 \mathrm{~g} /$ day) consumed more sodium than was reported for USA and UK general populations ( $3.6 \mathrm{~g} /$ day and $3.4 \mathrm{~g} /$ day, respectively) [22], and a cohort of diabetes patients in the US (2.5-3.4 g/day) [23]. However, consumption of sodium among the JDCS patients was lower than in the general Japanese population in 2001 (4.5 g/day) [24]. Further studies are needed to clarify whether the medical nutritional treatment that restricts sodium intake to values according to the USA and European guidelines would reduce incident CVD in the management of diabetes.

Several plausible findings suggest the reasons why diabetes complications develop regardless of sodium intake in elderly patients with type 2 diabetes. First, the relationship between sodium intake and the risk of arteriosclerosis is not linear. A previous prospective study in 17 countries reported that mortality and cardiovascular events are greater on both a high sodium excretion, as well as on a low sodium excretion [25], and some populations demonstrate an increase in blood pressure on a low sodium diet and are considered to be inverse salt sensitive [26]. Therefore, some patients with diabetes who are inverse salt-sensitive and follow national federal guidelines for lower sodium consumption may have elevated blood pressure by activation of renin-aldosterone and sympathetic nerve systems [27], leading to the development of vascular complications. Second, elderly type 2 diabetes patients with low sodium intake have lower intakes of food groups and nutrition that were reported to be associated with reduced risk of diabetic complications and mortality. Our current study showed that study participants with low sodium intake had a significantly lower intake of all food groups and nutritional than those with high sodium intake. Several previous prospective studies reported that higher intakes of polyunsaturated fatty acids (PUFA), carotene, vitamin B6, and vitamin C were associated with a lower risk of developing retinopathy in patients with diabetes [28-30]. This suggests that low intake of those nutrients, as well as foods related to these nutrients, is associated with an increased risk of retinopathy in the low sodium intake quartile. This may have counteracted the effect of low sodium intake in preventing the development of atherosclerosis via blood pressure control.

In addition, in our subgroup analysis, the trend for risk of developing retinopathy at six years was positively correlated with increasing sodium intake at baseline in patients with type 2 diabetes younger than 75 years, but there was no such trend in those older than 75 years. Therefore, the impact of nutrients on health outcomes may vary with age [31]. It has been reported that the risk of death is increased in those with type 2 diabetes older than 75 years who have low protein, vegetable, and fish intakes [32,33]. Elderly patients with type 2 diabetes should have their dietary intake monitored not only for excessive sodium intake, but also for intakes other food groups and nutrition intakes that occur with low sodium intake to avoid dietary habits that would lead to malnutrition, sarcopenia, frailty development of diabetic complications and death.

Aging is a major risk factor that can have a significant impact on advanced glycation and progression of atherosclerosis, which are the main causes of the development of diabetes complications. The main risk factor profiles of advanced glycation end products and atherosclerosis-mediated diabetes complications include sex, age, diabetes-specific and glycemia-related factors, including $\mathrm{HbA} 1 \mathrm{c}$, diabetes duration, BMI, systolic blood pressure, lipid levels, and current smoking [34-37]. These can result from a complex combination of pathological pathways, such as hyperglycemia itself, increased insulin resistance, inflammatory responses, and decreased fluidity of the cell membrane [38]. Excessive sodium intake leads to chronic hypertension, a major cause of atherosclerosis [39,40]. It was reported that the administration of salt induced significant insulin resistance through the production of oxidative stress in salt-sensitive hypertensive rats [41,42], and that high sodium intake predicted the risk of type 2 diabetes in a prospective cohort study [43]. Since our current study enrolled elderly patients with type 2 diabetes, it can be inferred 
that insulin resistance and atherosclerosis, due to aging greatly affected the development of diabetes complications in the participants in J-EDIT, and the effect of sodium intake on insulin resistance and atherosclerosis was slight, unlike that in the middle-aged and young-old patients with type 2 diabetes reported in the JDCS [6].

As to the subgroup analysis according to vegetable intake, among patients whose vegetable intake was less than the average of $268.7 \mathrm{~g}$, the HR for diabetic retinopathy in the top vs. the bottom quartile of sodium intake was significantly increased (3.70 (1.37-10.02), $p=0.010)$, but no significant association between sodium intake and diabetic retinopathy was observed in patients whose vegetable intake was equal to or higher than the participants' mean vegetable intake. As to the reason for the various results for vegetable intake, low vegetable intake contributes to an increased risk of developing diabetic retinopathy. Increased fruit and vegetable intake was associated with reduced incident diabetic retinopathy among patients with type 2 diabetes [44]. Additionally, it was shown that the risk for diabetic retinopathy declined with increased intake of vitamin $C$ and carotene, which are found in vegetables $[17,44]$. It can be speculated that the combination of low vegetable intake and high salt intake increased the risk of developing retinopathy in elderly patients with type 2 diabetes.

To the best of our knowledge, this is the first study on dietary sodium intake and the incidence of diabetes complications in which elderly patients with type 2 diabetes were prospectively registered based on $\mathrm{HbA1c}$ levels and not retrospectively selected based on self-reported diabetes status. Other strengths include treatment and follow-up plans that were conducted in institutes specializing in diabetes care and adjudication of cardiovascular events by an independent central committee.

The limitations of this study must be considered. First, the number of participants of our current study is limited to 912 though this study is conducted as a part of nationwide multicenter prospective study. In addition, this study investigated the association between sodium intake and diabetes complications in elderly patients with type 2 diabetes who had not developed any complications at the age of $65-85$ years. J-EDIT previously reported that the proportion of elderly patients with type 2 diabetes with ischemic heart disease, cerebrovascular disease, retinopathy, and microalbuminuria or persistent proteinuria were $16 \%$, $13 \%, 47 \%$, and $43 \%$, respectively [10]. Further studies are needed to examine the relationship between sodium intake and the severity and recurrence of diabetes complications in elderly patients with type 2 diabetes who had a history of diabetes complications. Second, the potential for bias, such as measurement errors in dietary assessments, confounding factors, and informative censoring, cannot be ruled out entirely. Third, as an observational study rather than a randomized trial, we could not conclude cause-effect relationships as to whether the medical nutritional treatment encouraging sodium reduction would reduce incident diabetic retinopathy in clinical practice. Moreover, it is not possible to say that sodium restriction is ineffective in elderly patients with type 2 diabetes. Fourth, our study did not observe any significant differences between blood pressure and dietary sodium intake. The percentage of patients treated by antihypertensive agents was similar in each quartile of dietary sodium intake. Given these results, a possible explanation may be that chronic high blood pressure could have been compensated for by increasing doses of antihypertensive drugs. Finally, our results may not be generally applicable to populations with different lifestyles or genetic factors. For example, the JDCS patients consumed a "highcarbohydrate low-fat" diet compared with Western patients with diabetes [45] and dietary sodium consumption in Japanese was generally higher than in Western people [22-24,45]. In addition, BMI and body weight are markedly different between patients in Japan and Western countries [46], and Asian patients have a much lower risk of CVD compared with Western patients and a higher risk of end-stage renal disease [47]. The contribution of such ethnic differences remains uncertain and is worthy of further research.

In conclusion, we found that high dietary sodium intake was not associated with the incidence of diabetic retinopathy, overt nephropathy, CVD, and all-cause mortality in elderly Japanese patients with type 2 diabetes. When these participants had a low 
vegetable intake, high sodium intake was associated with an elevated incidence of diabetic retinopathy. It was suggested that dietary salt restriction as a medical nutritional treatment for elderly patients with type 2 diabetes would be useful if consumption of vegetables is concomitantly encouraged.

Author Contributions: Conceptualization: H.I., H.S., and A.A.; data curation: C.H., R.A., S.T. (Sachiko Tanaka), C.K., S.T. (Shiro Tanaka), Y.Y., R.K. (Remi Kodera), K.F., R.K. (Ryo Kawasaki), T.M., H.Y., H.I., H.S., and A.A.; formal analysis: R.A. and S.T. (Shiro Tanaka); funding acquisition: H.I., and H.S.; investigation: C.H., R.A., S.T. (Shiro Tanaka), C.K., S.T. (Sachiko Tanaka), Y.Y., R.K. (Remi Kodera), K.F., R.K. (Ryo Kawasaki), T.M., H.Y., H.I., H.S., and A.A.; methodology: C.H., R.A., S.T. (Shiro Tanaka), H.S., and A.A.; project administration: H.I.; resources: Y.Y., H.S., and A.A.; software: R.A. and S.T. (Shiro Tanaka); supervision: H.S. and A.A.; validation: C.H., R.A., H.S., and A.A.; visualization: C.H., R.A., H.S., and A.A.; writing-original draft: C.H., R.A., H.S., and A.A.; writing-review and editing: C.H., R.A., S.T. (Shiro Tanaka), C.K., S.T. (Sachiko Tanaka), Y.Y., R.K. (Remi Kodera), K.F., R.K. (Ryo Kawasaki), T.M., H.Y., H.I., H.S., and A.A. H.S. is the guarantor of this work and, as such, had full access to all of the data in the study and takes responsibility for the integrity of the data and the accuracy of the data analysis. All authors have read and agreed to the published version of the manuscript.

Funding: This work was supported by the Ministry of Health, Labor, and Welfare and the Japan Foundation for Aging and Health, Japan. H.S. was supported by a Grant-in-Aid for Scientific Research (\#19H04028) from the Japan Society for the Promotion of Science (JSPS). The sponsor had no role in the design and conduct of the study; collection, management, analysis, and interpretation of the data; or preparation, review, or approval of the manuscript.

Institutional Review Board Statement: The study was conducted according to the guidelines of the Declaration of Helsinki, and approved by the Ethics Committees of of Tokyo Metropolitan Tama Geriatric Hospital (protocol code TMTGH-R1, date of approval 2001-2-3) and Tokyo Metropolitan Geriatric Hospital (protocol code TMGH2001-R74, date of approval 2001-5-9).

Informed Consent Statement: Informed consent was obtained from all subjects involved in the study.

Data Availability Statement: The data presented in this study are available on request from the corresponding author. The data are not publicly available due to privacy of participants.

Acknowledgments: We thank all of the patients, staff members, and diabetologists all over Japan for their long-standing collaboration in this study. Thanks are extended to Mami H who took part in the Japanese Diabetes Complications Study and the Japanese Elderly Diabetes Intervention Trial. The authors thank the participants who took part in the study. We also thank Mami Haga and -Natsuko Tada, Niigata University, for their excellent secretarial assistance.

Conflicts of Interest: The authors declare no conflict of interest. The research organizations providing funding support did not have any role in the design and conduct of the study; in the correction, management, analysis, and interpretation of the data; or in the preparation, review, or approval of the manuscript.

\section{References}

1. American Diabetes Association. 5. Lifestyle Management: Standards of Medical Care in Diabetes-2019. Diabetes Care 2019, 42, S46-S60. [CrossRef] [PubMed]

2. Mann, J.I.; De Leeuw, I.; Hermansen, K.; Karamanos, B.; Karlstrom, B.; Katsilambros, N.; Riccardi, G.; Rivellese, A.A.; Rizkalla, S.; Slama, G.; et al. Evidence-based nutritional approaches to the treatment and prevention of diabetes mellitus. Nutr. Metab. Cardiovasc. Dis. 2004, 14, 373-394. [CrossRef]

3. Araki, E.; Goto, A.; Kondo, T.; Noda, M.; Noto, H.; Origasa, H.; Osawa, H.; Taguchi, A.; Tanizawa, Y.; Tobe, K.; et al. Japanese Clinical Practice Guideline for Diabetes 2019. Diabetol. Int. 2020, 11, 165-223. [CrossRef]

4. Thomas, M.C.; Moran, J.; Forsblom, C.; Harjutsalo, V.; Thorn, L.; Ahola, A.; Wadén, J.; Tolonen, N.; Saraheimo, M.; Gordin, D.; et al. The Association between Dietary Sodium Intake, ESRD, and All-Cause Mortality in Patients with Type 1 Diabetes. Diabetes Care 2011, 34, 861-866. [CrossRef]

5. Ekinci, E.I.; Clarke, S.; Thomas, M.C.; Moran, J.L.; Cheong, K.; MacIsaac, R.J.; Jerums, G. Dietary salt intake and mortality in patients with type 2 diabetes. Diabetes Care 2011, 34, 703-709. [CrossRef] 
6. Horikawa, C.; Yoshimura, Y.; Kamada, C.; Tanaka, S.; Tanaka, S.; Hanyu, O.; Araki, A.; Ito, H.; Tanaka, A.; Ohashi, Y.; et al. Dietary sodium intake and incidence of diabetes complications in Japanese patients with type 2 diabetes: Analysis of the Japan Diabetes Complications Study (JDCS). J. Clin. Endocrinol. Metab. 2014, 99, 3635-3643. [CrossRef]

7. Saulnier, P.; Gand, E.; Ragot, S.; Bankir, L.; Piguel, X.; Fumeron, F.; Rigalleau, V.; Halimi, J.; Marechaud, R.; Roussel, R.; et al. Urinary Sodium Concentration Is an Independent Predictor of All-Cause and Cardiovascular Mortality in a Type 2 Diabetes Cohort Population. J. Diabetes. Res. 2017, 2017, 5327352. [CrossRef] [PubMed]

8. Sone, H.; Tanaka, S.; Iimuro, S.; Tanaka, S.; Oida, K.; Yamasaki, Y.; Oikawa, S.; Ishibashi, S.; Katayama, S.; Yamashita, H.; et al. Long-term lifestyle intervention lowers the incidence of stroke in Japanese patients with type 2 diabetes: A nationwide multicentre randomised controlled trial (the Japan Diabetes Complications Study). Diabetologia 2010, 53, 419-428. [CrossRef] [PubMed]

9. Tanaka, S.; Tanaka, S.; Iimuro, S.; Yamashita, H.; Katayama, S.; Ohashi, Y.; Akanuma, Y.; Yamada, N.; Sone, H. On behalf of the Japan Diabetes Complications Study Group. Cohort profile: The Japan diabetes complications study: A long-term follow-up of a randomised lifestyle intervention study of type 2 diabetes. Int. J. Epidemiol. 2014, 43, 1054-1062. [CrossRef]

10. Araki, A.; Iimuro, S.; Sakurai, T.; Umegaki, H.; Iijima, K.; Nakano, H.; Oba, K.; Yokono, K.; Sone, H.; Yamada, N.; et al. Long-term multiple risk factor interventions in Japanese elderly diabetic patients: The Japanese Elderly Diabetes Intervention Trial—Study design, baseline characteristics and effects of intervention. Geriatr. Gerontol. Int. 2012, 12, 7-17. [CrossRef] [PubMed]

11. Kalyani, R.R.; Golden, S.H.; Cefalu, W.T. Diabetes and Aging: Unique Considerations and Goals of Care. Diabetes Care 2017, 40, 440-443. [CrossRef]

12. International Diabetes Federation. IDF DIABETES ATLAS Ninth Edition 2019. Available online: https://diabetesatlas.org/en/ resources / (accessed on 20 December 2020).

13. United Nations, Department of Economic and Social Affairs, Population Division. World Population Prospects 2019: Highlights (ST/ESA/SER.A/423). 2019. Available online: https://population.un.org/wpp/Publications/Files/WPP2019_Highlights.pdf (accessed on 20 December 2020).

14. World Health Organization. Report of a World Health Organization Consultation. Use of glycated haemoglobin (HbA1c) in the diagnosis of diabetes mellitus. Diabetes Res. Clin. Pract. 2011, 93, 299-309. [CrossRef]

15. The Committee of Japan Diabetes Society on the Diagnostic Criteria of Diabetes Mellitus. Report of the committee on the classification and diagnostic criteria of diabetes mellitus. J. Diabetes Investig. 2010, 1, 212-228. [CrossRef]

16. Takahashi, K.; Yoshimura, Y.; Kaimoto, T.; Kunii, D.; Komatsu, T.; Yamamoto, S. Validation of a Food Frequency Questionnaire based on food groups for estimating individual nutrient intake. Jpn. J. Nutr. 2001, 59, 221-232. [CrossRef]

17. Ministry of Education, Culture, Sports, Science and Technology, Japan. Standard Tables of Food Composition in Japan 2005. Available online: http:/ / www.mext.go.jp/b_menu/shingi/gijyutu/gijyutu3/toushin/05031802.htm (accessed on 20 December 2020).

18. Beaglehole, R.; Stewart, A.W.; Butler, M. Comparability of old and new World Health Organization criteria for definite myocardial infarction. Int. J. Epidemiol. 1987, 16, 373-376. [CrossRef]

19. Tuomilehto, J.; Kuulasmaa, K. WHO MONICA Project: Assessing CHD mortality and morbidity. Int. J. Epidemiol. 1989, 18, S38-S45. [PubMed]

20. The Committee of Ministry of Health. Labor and Welfare on the Diagnostic Criteria of Stroke. Report of the Committee of Ministry of Health, Labor and Welfare on the Diagnostic Criteria of Stroke; Ministry of Health, Labor and Welfare: Tokyo, Japan, 2013.

21. Aho, K.; Harmsen, P.; Hatano, S.; Marquardsen, J.; Smirnov, V.E.; Strasser, T. Cerebrovascular disease in the community: Results of a WHO collaborative study. Bull. World Health Organ. 1980, 58, 113-130. [PubMed]

22. Anderson, C.A.M.; Appel, L.J.; Okuda, N.; Brown, I.J.; Chan, Q.; Zhao, L.; Ueshima, H.; Kesteloot, H.; Miura, K.; Curb, J.D.; et al. Dietary sources of sodium in China, Japan, the United Kingdom, and the United States, women and men aged 40 to 59 years: The INTERMAP Study. J. Am. Diet. Assoc. 2010, 110, 736-745. [CrossRef]

23. Eilat-Adar, S.; Xu, J.; Zephier, E.; O'Leary, V.; Howard, B.V.; Resnick, H.E. Adherence to dietary recommendations for saturated fat, fiber, and sodium is low in American Indians and other U.S. adults with diabetes. J. Nutr. 2008, 138, 1699-1704. [CrossRef] [PubMed]

24. National Institute of Health and Nutrition. National Health and Nutrition Survey $>$ National Health and Nutrition Survey results: Nutritional Intake Status Survey. Available online: https://www.nibiohn.go.jp/eiken/kenkounippon21/en/eiyouchousa/ kekka_eiyou_chousa_nendo_noniacin.html (accessed on 20 December 2020).

25. O’Donnell, M.; Mente, A.; Rangarajan, S.; McQueen, M.J.; Wang, X.; Liu, L.; Yan, H.; Lee, S.F.; Mony, P.; Devanath, A.; et al Urinary sodium and potassium excretion, mortality, and cardiovascular events. N. Engl. J. Med. 2014, 371, 612-623. [CrossRef] [PubMed]

26. Felder, R.A.; White, M.J.; Williams, S.M.; Jose, P.A. Diagnostic tools for hypertension and salt sensitivity testing. Curr. Opin. Nephrol. Hypertens. 2013, 22, 65-76. [CrossRef] [PubMed]

27. Wang, J.; Deng, Y.; Zou, X.; Luo, H.; Jose, P.A.; Fu, C.; Yang, J.; Zeng, C. Long-term low salt diet increases blood pressure by activation of the renin-angiotensin and sympathetic nervous systems. Clin. Exp. Hypertens. 2019, 41, 739-746. [CrossRef] [PubMed]

28. Dow, C.; Mancini, F.; Rajaobelina, K.; Boutron-Ruault, M.; Balkau, B.; Bonnet, F.; Fagherazzi, G. Diet and risk of diabetic retinopathy: A systematic review. Eur. J. Epidemiol. 2018, 33, 141-156. [CrossRef] [PubMed] 
29. Wong, M.Y.Z.; Man, R.E.K.; Fenwick, E.K.; Gupta, P.; Li, L.; van Dam, R.M.; Chong, M.F.; Lamoureux, E.L. Dietary intake and diabetic retinopathy: A systematic review. PLOS ONE 2018, 13, e0186582. [CrossRef] [PubMed]

30. Horikawa, C.; Aida, R.; Kamada, C.; Fujihara, K.; Tanaka, S.; Tanaka, S.; Araki, A.; Yoshimura, Y.; Moriya, T.; Akanuma, Y.; et al. Vitamin B6 intake and incidence of diabetic retinopathy in Japanese patients with type 2 diabetes: Analysis of data from the Japan Diabetes Complications Study (JDCS). Eur. J. Nutr. 2020, 59, 1585-1594. [CrossRef]

31. Tamura, Y.; Omura, T.; Toyoshima, K.; Araki, A. Nutrition Management in Older Adults with Diabetes: A Review on the Importance of Shifting Prevention Strategies from Metabolic Syndrome to Frailty. Nutrients 2020, 12, 3367. [CrossRef]

32. Yamaoka, T.; Araki, A.; Tamura, Y.; Tanaka, S.; Fujihara, K.; Horikawa, C.; Aida, R.; Kamada, C.; Yoshimura, Y.; Moriya, T.; et al. Association between low protein intake and mortality in patients with type 2 diabetes. Nutrients 2020, 12, 1629. [CrossRef]

33. Iimuro, S.; Yoshimura, Y.; Umegaki, H.; Sakurai, T.; Araki, A.; Ohashi, Y.; Iijima, K.; Ito, H.; Japanese Elderly Diabetes Intervention Trial Study Group. Dietary pattern and mortality in Japanese elderly patients with type 2 diabetes mellitus: Does a vegetableand fish-rich diet improve mortality? An explanatory study. Geriatr. Gerontol. Int. 2012, 12, 59-67. [CrossRef] [PubMed]

34. Stevens, R.J.; Kothari, V.; Adler, A.I.; Stratton, I.M.; United KingdomProspective Diabetes Study (UKPDS) Group. The UKPDS risk engine: A model for the risk of coronary heart disease in type II diabetes (UKPDS 56). Clin. Sci. (Lond.) 2001, 101, 671-679. [CrossRef]

35. Balkau, B.; Hu, G.; Qiao, Q.; Tuomilehto, J.; Borch-Johnsen, K.; Pyörälä, K.; DECODE Study Group; European Diabetes Epidemiology Group. Prediction of the risk of cardiovascularmortality using a score that includes glucose as a risk factor. Diabetologia 2004, 47, 2118-2128. [PubMed]

36. Cederholm, J.; Eeg-Olofsson, K.; Eliasson, B.; Zethelius, B.; Nilsson, P.M.; Gudbjörnsdottir, S.; Swedish National Diabetes Register. Risk prediction of cardiovascular disease in type 2 diabetes: A risk equation from the Swedish National Diabetes Register. Diabetes Care 2008, 31, 2038-2043. [CrossRef]

37. Tanaka, S.; Tanaka, S.; Iimuro, S.; Yamashita, H.; Katayama, S.; Akanuma, Y.; Yamada, N.; Araki, A.; Ito, H.; Sone, H.; et al. Predicting macro- and microvascular complications in type 2 diabetes: The Japan Diabetes Complications Study/the Japanese Elderly Diabetes Intervention Trial risk engine. Diabetes Care 2013, 36, 1193-1199. [CrossRef]

38. Forbes, J.M.; Cooper, M.E. Mechanisms of diabetic complications. Physiol. Rev. 2013, 93, 137-188. [CrossRef] [PubMed]

39. Adrogué, H.J.; Madias, N.E. Sodium and potassium in the pathogenesis of hypertension. N. Engl. J. Med. 2007, 356, 1966-1978. [CrossRef]

40. Takahashi, H.; Yoshika, M.; Komiyama, Y.; Nishimura, M. The central mechanism underlying hypertension: A review of the roles of sodium ions, epithelial sodium channels, the renin-angiotensin-aldosterone system, oxidative stress and endogenous digitalis in the brain. Hypertens. Res. 2011, 34, 1147-1160. [CrossRef] [PubMed]

41. Ogihara, T.; Asano, T.; Ando, K.; Sakoda, H.; Anai, M.; Shojima, N.; Ono, H.; Onishi, Y.; Fujishiro, M.; Abe, M.; et al. High-salt diet enhances insulin signaling and induces insulin resistance in Dahl salt-sensitive rats. Hypertension 2002, 40, 83-89. [CrossRef] [PubMed]

42. Han, S.; Cheng, D.; Liu, N.; Kuang, H. The relationship between diabetic risk factors, diabetic complications and salt intake. J. Diabetes Complicat. 2018, 32, 531-537. [CrossRef]

43. Hu, G.; Jousilahti, P.; Peltonen, M.; Lindström, J.; Tuomilehto, J. Urinary sodium and potassium excretion and the risk of type 2 diabetes: A prospective study in Finland. Diabetologia 2005, 48, 1477-1483. [CrossRef] [PubMed]

44. Tanaka, S.; Yoshimura, Y.; Kawasaki, R.; Kamada, C.; Tanaka, S.; Horikawa, C.; Ohashi, Y.; Araki, A.; Ito, H.; Akanuma, Y.; et al. Fruit intake and incident diabetic retinopathy with type 2 diabetes. Epidemiology 2013, 24, 204-211. [CrossRef]

45. Horikawa, C.; Yoshimura, Y.; Kamada, C.; Tanaka, S.; Tanaka, S.; Takahashi, A.; Hanyu, H.; Araki, A.; Ito, H.; Tanaka, A.; et al. Dietary intake in Japanese patients with type 2 diabetes: Analysis from Japan Diabetes Complications Study. J. Diabetes Investig. 2014, 5, 176-187. [CrossRef]

46. Sone, H.; Ito, H.; Ohashi, Y.; Akanuma, Y.; Yamada, N.; Japan Diabetes Complication Study Group. Obesity and type 2 diabetes in Japanese patients. Lancet 2003, 361, 85. [CrossRef]

47. Karter, A.J.; Ferrara, A.; Liu, J.Y.; Moffet, H.H.; Ackerson, L.M.; Selby, J.V. Ethnic disparities in diabetic complications in an insured population. JAMA 2002, 287, 2519-2527. [CrossRef] [PubMed] 